\title{
Solar Simulated Radiation Induced Cell Death Depends on Spectral Distribution and Irradiance But Not Output Delivery
}

\author{
Alanna Maguire \\ Technological University Dublin \\ Fiona Lyng \\ Technological University Dublin, fiona.lyng@tudublin.ie \\ James Walsh \\ Technological University Dublin, james.walsh@tudublin.ie
}

Follow this and additional works at: https://arrow.tudublin.ie/radart

Part of the Radiology Commons

\section{Recommended Citation}

Maguire, A., Lyng, F.M. \& Walsh, J.E. (2010) Solar simulated radiation induced cell death depends on spectral distribution and irradiance but not output delivery. Radiat Prot Dosimetry. 140(2) pp.147-57. doi:10.1093/rpd/ncq080

This Article is brought to you for free and open access by the Radiation and Environmental Science Centre at ARROW@TU Dublin. It has been accepted for inclusion in Articles by an authorized administrator of ARROW@TU Dublin. For more information, please contact arrow.admin@tudublin.ie, aisling.coyne@tudublin.ie, gerard.connolly@tudublin.ie.

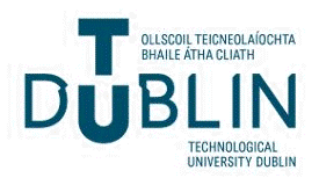




\title{
SOLAR SIMULATED RADIATION INDUCED CELL DEATH DEPENDS ON SPECTRAL DISTRIBUTION AND IRRADIANCE BUT NOT OUTPUT DELIVERY
}

\author{
Alanna Maguire ${ }^{1}$, Fiona M. Lyng ${ }^{1}$, James E. Walsh², \\ ${ }^{1}$ Radiation and Environmental Science Centre (RESC), Focas Institute, Dublin Institute of Technology, Camden \\ Row, Dublin 8, Ireland \\ ${ }^{2}$ Department of Physics, Dublin Institute of Technology, Kevin Street, Dublin 8, Ireland
}

Photo biological investigations are dependent on calibration and characterisation to determine the relevance of an artificial irradiator to the study at hand. The importance of this has been voiced in the literature. However, the importance of output delivery is relatively unknown. The biological relevance of a high energy, rapidly pulsing solar simulator was investigated using the clonogenic assay and was found to be reciprocity law compliant despite an exaggerated $U V$ irradiance in excess of $1600 \mathrm{Wm}^{-2}$ delivered per pulse. In fact, it was found to be the least cytotoxic irradiator compared to a second solar simulator and a UVB fluorescent lamp with continuous $\mathrm{UV}$ irradiances of $55 \mathrm{Wm}^{-2}$ and $6.4 \mathrm{Wm}^{-2}$ respectively. The reduced survival observed with the continuous irradiators is attributed to differences in spectral irradiance and distribution, particularly in the UVB, which in the absence of thorough calibration and characterisation may have resulted in erroneous conclusions. 


\section{INTRODUCTION}

Skin cancer is a globally growing epidemic whose incidence is known to be related to ultraviolet (UV) radiation exposure ${ }^{(1-5)}$. Although exposure to $\mathrm{UV}$ is highly variable within a population due to individual habits (frequency of exposure, use of artificial tanning beds) and behaviour (use of protection), UV from solar radiation is known to be the main environmental factor contributing to the formation of these malignancies.

When performing biological irradiation experiments, the ideal situation would be to utilise solar radiation to elicit the biological response under investigation. However, this is rarely a viable option due to the stringent aseptic conditions required for cell culture and the daily fluctuations in spectral irradiance experienced with solar radiation due to weather conditions, time of day, season and geographical location. Stability and reproducibility are essential to achieve trustworthy data and it is for this reason that employing an artificial irradiator is the most practical option. Although artificial irradiators are the more pragmatic alternatives to solar radiation, they are not without their challenges. The type of source employed in radiation studies can vary immensely from the type of source, wavelength range, spectral distribution, spectral irradiance and delivery (continuous versus non continuous) of the lamp ${ }^{(6,7)}$ each with different advantages and disadvantages over one another.

A plethora of studies exist in the literature looking at the biological effects of UV. The majority of work to date that has provided vital information regarding the ability of UV to elicit detrimental effects (erythma, mutagenesis, immunosuppression, oxidative stress and initiation of skin carcinogenesis ${ }^{(8-14)}$ ) and positive effects (vitamin D synthesis, skin disorder treatment ${ }^{(3,15)}$ ) have been determined through the use of fluorescent sunlamps. The information obtained from such irradiators has provided the foundations of solar radiation investigations. However for more detailed studies on radiation induced impairment of cellular functions, attention must turn to the instrumentation employed and their relevance to the study at hand.

Wavelength and energy are inversely proportional, where the efficacy of radiation to elicit a biological effect with decreasing wavelength is governed not only by the relative spectral effectiveness of radiation $^{(16,17)}$ and biological action spectra ${ }^{(18,19)}$ but also the penetrative capacity of radiation to reach its potential targets. This combined with the ability of a source to administer environmentally relevant emissions as would be experienced at the terrestrial level stresses the importance of spectral distribution and irradiance of a given source when attempting to elucidate the mechanisms involved in skin carcinogenesis.

*Corresponding author: alanna.maguire@dit.ie
In addition to the spectral distribution and irradiance of an artificial source, the manner in which the output of an irradiator is administered is another aspect of a source that may have important implications for photo biological investigations. The Bunsen Roscoe or reciprocity law states that all photochemical reactions are dependent on the total energy absorbed irrespective of the manner in which the dose is delivered ${ }^{(20,21)}$ however reservations exist regarding the applicability of this law to biological samples ${ }^{(22)}$ due to the highly complex response of cells to a given stimulus. Since solar radiation is a continuous source that varies in spectral irradiance but not delivery, the nature of an artificial source may also have significant implications regarding biological and environmental relevance.

To investigate the above characteristics and their possible effects on biological samples, four artificial sources were employed for this study, two xenon arc solar simulators and two fluorescent UV lamps. Each source was thoroughly characterised, using spectroradiometry to obtain the spectral distribution and irradiance for each source while their output behaviour was determined using a calibrated GaAsP photodiode. Once characterised, each source was employed to perform photo biological experiments on a human keratinocyte cell line, HaCaT cells, to determine the ability of each irradiator to incur cell death as measured using the clonogenic assay.

\section{MATERIALS AND METHODS}

\section{Dosimetry}

To determine the spectral distribution and irradiance of the different irradiators, a miniature USB2000 fibre optic spectrometer (Ocean Optics, Dunedin FL, USA) was employed to perform spectroradiometry. Radiation is coupled to the 50 $\mu \mathrm{m}$ input slit of the spectrometer via a $600 \mu \mathrm{m}$ fibre optic. A CC-3-UV PTFE cosine corrected diffuser (Ocean Optics, Dunedin FL, USA) with a response over $200-1100 \mathrm{~nm}^{(23)}$ was mounted at the front end of the fibre optic to integrate any variations in spatial distribution of each irradiator at its respective calibration point over a $2 \pi$ field of view. Radiation collected by the input optics is dispersed by a fixed grating (600 lines $/ \mathrm{mm}$ ) across a 2048 pixel linear CCD array with a resulting FWHM resolution of $1.5 \mathrm{~nm}$. The spectrometer was calibrated using a Bentham CL6h spectral irradiance standard (Bentham instruments Ltd, Berkshire, UK), which has a spectral irradiance from 250 to 3000 $\mathrm{nm}$ and is traceable to National Physical Laboratory standards (NPL, Teddington, UK). Each source was sampled using the USB2000 spectrometer with an integration time of $100 \mathrm{~ms}$ per scan to achieve maximum signal without saturation and an average of 200 scans to increase the signal to noise ratio ${ }^{(24)}$. Although the USB2000 has been reported to possess poor stray light rejection ${ }^{(25)}$, it was also shown that with adequate correction that the levels of stray light could be significantly reduced to less then $1 \%$ at $250 \mathrm{~nm}^{(25)}$. A simple correction analogous to 
background subtraction was employed in this study where the difference in signal between the blacked out pixels corresponding to $180 \mathrm{~nm}$ and at $250 \mathrm{~nm}$, a wavelength at which no signal would be expected, was determined and subtracted at all wavelengths. This was performed for each independent sampling due to the possibility of differing stray light profiles with each sampling. The irradiance of each source was relatively uniform, varying less then $10 \%$ across the exposure field at which cells would be located during irradiation.

Once calibrated, the spectrometer was employed to obtain the calibrated spectral distribution $\left(\mathrm{Wm}^{-2}\right)$ at the exposure level for all sources employed, the Qsun solar simulator (Q-panel, Cleveland, USA), the Oriel solar simulator (Oriel-Newport, California, USA), a UVA fluorescent lamp (Ultra Violet products (UVP) Ltd Cambridge UK) and a UVB fluorescent lamp (UVItec, Cambridge UK). While the spectral irradiance of both solar simulators is equivalent to that for typical air mass (AM) 1 at their respective calibration points, their temporal outputs are different to one another. The Q-sun is a non continuous $100 \mathrm{~Hz}$ pulsed xenon arc lamp while the Oriel xenon arc lamp is a continuous source. The difference in the output delivery (continuous versus pulsed/non continuous) of the two solar simulators permitted an investigation into whether cells irradiated using the Q-sun were overly stressed or damaged by the high irradiance, high frequency pulsed delivery or whether the spectral distribution delivered per second was the key factor. The spectral distribution of the fluorescent lamps were found to be significantly different to that of the solar simulators, allowing us to investigate the effects of dose delivery and spectral distribution for typical solar irradiation levels or for lamps commonly used in cell dosimetry research. The UVA-B spectral intensities were computed by integrating under the curve for each artificial source using waveband intervals of $280-315 \mathrm{~nm}$ and $315-400 \mathrm{~nm}$ for the UVB and UVA respectively ${ }^{(26)}$. Exposures are presented in terms of time but can be converted to UV dose using the fact that $1 \mathrm{Wm}^{-2}$ equals $1 \mathrm{Jm}^{-2} \mathrm{~s}^{-1}$. For example, a 10 minute (600 second) exposure with an irradiator that provides a UV irradiance of $63.6 \mathrm{Wm}^{-2}\left(63.6 \mathrm{Jm}^{-2} \mathrm{~s}^{-1}\right)$ provides a dose of 38,160 $(63.6 \times 600) \mathrm{Jm}^{-2}$ or $3.816 \mathrm{Jcm}^{-2}$.

\section{Cell culture}

A human keratinocyte cell line, HaCaT cells, was used for this study. HaCaT cells are immortal but non-malignant with a doubling time of 21 hours $^{(27)}$ and mutations in both p53 alleles ${ }^{(28)}$. HaCaT cells were cultured in Dulbecco's MEM-F12 (1:1) medium (Cambrex, U.K.) containing 10\% fetal calf serum (Gibco, Irvine, U.K.), $1 \%$ penicillinstreptomycin solution 1000IU (Gibco, Irvine, U.K.) and $1 \mu \mathrm{g} / \mathrm{ml}$ hydrocortisone (Sigma, Dorset, U.K.). Cells were incubated under humid conditions at $37^{\circ} \mathrm{C}$, with $5 \% \mathrm{CO}_{2}$ in air. Subculture was routinely performed when cells were 80-90\% confluent, using a $1: 1$ solution of $0.2 \%$ trypsin and $1 \mathrm{mM}$ versene at $37^{\circ} \mathrm{C}$. Once detached, the trypsin was neutralised and the cells centrifuged, after which the neutralised trypsin/versene solution was decanted and the pellet re-suspended in fresh cell culture medium and a single cell suspension obtained. Cell counts were determined using a Haemocytometer.

The HaCaT cell line has a plating efficiency of 27.2 $\pm 3.6 \%$. In order to obtain a reasonable number of colonies, cells were seeded at 400 cells in $3 \mathrm{ml}$ cell culture medium (DMEM-F12) per well in Nunclon 6 well plates and incubated (conditions as above) overnight (16 hours) prior to irradiation. The extended incubation period between seeding and irradiation was employed for all experiments since PBS exposures required cell washes which resulted in reduced and irregular control colony numbers compared to non washed controls when insufficient time for attachment was allowed. However dishes were checked prior to irradiation to ensure that the plated cells had not divided and that they were still single cells.

\section{Exposures}

The exposure field of each irradiator was disinfected using Virkon solution and then sterilised using $100 \%$ methanol prior to irradiation enabling exposures to be performed without lids to eliminate attenuation effects of the spectral irradiance due to the plastic. After the appropriate time interval, the lid was replaced and the cells removed from the exposure field and returned to the incubator for 7 days before survival was assayed using the clonogenic assay. Controls were handled identically but received sham irradiation.

\section{Direct DMEM-F12 exposures}

Cells were seeded and exposed as outlined above with no medium change before or after exposures.

\section{Direct PBS exposures}

Cells were seeded and exposed as outlined above. However prior to exposure, DMEM-F12 was harvested, filtered and incubated, cells were washed once with $1 \mathrm{ml}$ pre warmed $\left(37^{\circ} \mathrm{C}\right) \mathrm{PBS}$ to remove traces of DMEM-F12, the wash discarded and the cells exposed in $3 \mathrm{ml}$ fresh pre warmed PBS. Post exposure, the exposed PBS was removed and discarded and the incubated DMEM-F12 replaced before cells were returned to the incubator for 7 days.

\section{Clonogenic assay}

Clonogenic expansion of single cells was determined using the method devised by Puck and Marcus (29). This method allows survival to be quantified post exposure to some toxic event by the ability of single cells to form macroscopic colonies distinguishable from one another, where such a colony is said to be a group of approximately 50 or more cells which should arise from a single cell.

With a doubling time of 21 hours, a 7 day incubation period is sufficient for $\mathrm{HaCaT}$ cells to form macroscopic colonies. Thus following 
treatment, cells were incubated for 7 days, after which cells were stained using a $20 \%$ carbol fuchsin solution for 5 minutes and scored. Survival curves were determined from the data collected.

\section{Statistics}

Results represent 3 independent tests, normalised to controls and presented as the mean $+/$ - the standard deviation. Statistical analyses of variance (ANOVA) were performed on the linear regressions and pairwise data using the Bonferroni adjustment were obtained. All analyses were done using statistical software package SAS 9.1 and SAS enterprise guide 3.0. Significance was taken at a level of $\mathrm{p} \leq 0.05$.

\section{RESULTS AND DISCUSSION}
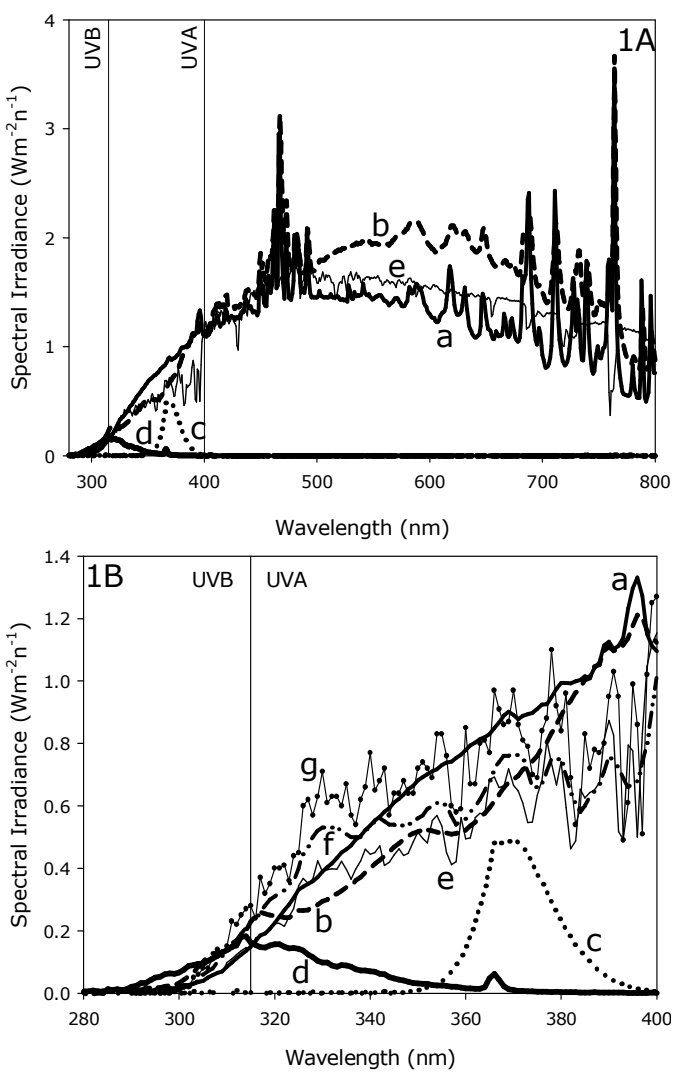

Figure 1 Spectral irradiance for the Q-sun solar simulator $(\mathrm{a},-$, the Oriel solar simulator operating at $35 \mathrm{~mA}$ (b,ーーーー), the UVA fluorescent lamp (c, $\cdots \cdots)$, and the UVB fluorescent lamp (d, a) at their respective exposure levels shown alongside solar irradiance sampled midsummer in Naples Italy $40^{\circ} \mathrm{N}(\mathrm{e},-\longrightarrow)$ from $280 \mathrm{~nm}$ to $800 \mathrm{~nm}$ in $(1 \mathrm{~A})$ and $280 \mathrm{~nm}$ to $400 \mathrm{~nm}$ in (1B). Also shown in (1B) are the irradiance spectra for solar radiation sampled midsummer in Albuquerque $38^{\circ} \mathrm{N}\left(\mathrm{f}, \boldsymbol{-} \cdot \boldsymbol{\sim}^{-}\right.$) and Melbourne $38^{\circ} \mathrm{S}(\mathrm{g},-)^{(30)}$.

The objective of this study was to assess the environmental relevance of four different irradiators. Each irradiator was characterised to ascertain reasonable approximations on their distribution spectra and spectral irradiance using spectroradiometry, which has been promoted in the literature as being the best and most effective manner in which to communicate the output of an irradiator unambiguously ${ }^{(30)}$. Once calibrated, the ability of each irradiator to incur cell death as determined by the clonogenic assay was assessed. The reliability of photo-biological data in the absence of sufficient dosimetry has been questioned in the literature ${ }^{(31)}$. Thus, the purpose of this study was not only to investigate the abilities of each irradiator but also to demonstrate how spectroradiometric data can aid photo-biological investigations by identifying environmentally irrelevant emissions, lethal or innocuous, and thereby reducing the probability of erroneous conclusions.

The calibrated spectra for all four irradiators are shown together over $280 \mathrm{~nm}$ to $800 \mathrm{~nm}$ in figure 1A and $280 \mathrm{~nm}$ to $400 \mathrm{~nm}$ in figure $1 \mathrm{~B}$. To demonstrate the ability of each artificial source to replicate solar radiation, the spectral distribution for solar radiation sampled in Naples, Italy $\left(40^{\circ} \mathrm{N}, 12\right.$ noon, July $\left.11^{\text {th }}\right)$ using the same spectroradiometry system used to sample all artificial sources is also shown in figures $1 \mathrm{~A}$ and 1B. To demonstrate agreement with the literature additional solar UV spectra provided by Diffey et $\mathrm{al}^{(30)}$ that correspond to Albuquerque, USA $\left(38^{\circ} \mathrm{N}\right.$, noon, July $\left.3^{\text {rd }}\right)$ and Melbourne, Australia $\left(38^{\circ} \mathrm{S}\right.$, solar noon, January $\left.17^{\text {th }}\right)$ in their respective summer seasons are also shown in figure 1B. The UV irradiances for all sources, artificial and natural, are listed in table 1 .

Both the Q-sun and Oriel solar simulators provide excellent representations of solar radiation as shown in figures $1 \mathrm{~A}$ and $1 \mathrm{~B}$. From the data presented in table 1, it can be seen that the Q-sun and Oriel administer UV irradiances of $63.6 \mathrm{Wm}^{-2}(\sim 62.3$ $\mathrm{Wm}^{-2}$ in the UVA and $\sim 1.3 \mathrm{Wm}^{-2}$ in the UVB) and $54.9 \mathrm{Wm}^{-2}\left(\sim 52.7 \mathrm{Wm}^{-2}\right.$ in the UVA and $\sim 2.2 \mathrm{Wm}^{-2}$ in the UVB) respectively. Figure $1 \mathrm{~B}$ shows the Oriel to have an interpretation closer to solar radiation over the UVA region compared to the simpler approximation of the Q-sun, which accounts for the majority of the differences observed in their spectral irradiance. However, these differences, when compared to the variations observed in solar radiation, as demonstrated in table 1 and in the literature ${ }^{(30,32)}$, are not unreasonable. Thus although neither solar simulator at their experimental working distances appear to mimic a geographic location exactly, both yield spectral intensities that can be experienced at the terrestrial level thus verifying the environmental relevance of each simulator.

The UVA fluorescent lamp can be seen to possess a spectral distribution that does not resemble that of solar radiation as shown in figures $1 \mathrm{~A}$ and $1 \mathrm{~B}$. Furthermore, integration of the curve yields an irradiance of just $9.49 \mathrm{Wm}^{-2}$ in the UVA and a negligible output in the UVB when sampled at the shortest working distance feasible $(12 \mathrm{~cm})$ for irradiation experiments. This UVA irradiance, as can be seen from table 1, is 5-6 fold less than the UVA spectral irradiance administered by the solar simulators. However, it is comparable to the UVA spectral irradiance modelled for Dublin, Ireland, on the winter solstice $\left(7.78 \mathrm{Wm}^{-2}\right.$ in the UVA, 0.01 
$\mathrm{Wm}^{-2}$ in the UVB, solar elevation of $13^{\circ}$ and air mass (AM) value of 4.45), using the Bird and Riordan mathematical model ${ }^{(33)}$. A solar radiation exposure at this latitude during the winter would not be expected to produce detrimental photo-biological effects. Similarly, none were expected from the UVA fluorescent lamp, irrespective of differences in spectral distribution, as was confirmed by photo biological experiments (data not shown).

The spectral irradiance of the UVB lamp was computed to be $3.96 \mathrm{Wm}^{-2}$ in the UVA and 2.43 $\mathrm{Wm}^{-2}$ in the UVB. Although the majority of the output lies in the UVA waveband region, UVB is the more biologically active waveband, thus such an emission is not expected to be confounding. From the data presented in table 1 , it can be seen that the UVB lamp possesses the largest UVB spectral irradiance of all artificial irradiators employed. However comparing it to the solar UVB spectral irradiance of $2.84 \mathrm{Wm}^{-2}$ for Singapore during an equinox (solar noon, $1^{\circ} \mathrm{N}$, solar elevation of $89^{\circ}$ and $\mathrm{AM}$ value of 1) as modelled by Garland et $\mathrm{al}^{(32)}$ establishes the environmental relevance of such a UVB irradiance. The spectral distribution of the UVB lamp, however, undermines its environmental relevance.

Close examination of figure $1 \mathrm{~B}$ shows that the spectral distribution of the UVB lamp does not reduce toward zero at $300 \mathrm{~nm}$ as is the case for solar radiation and both solar simulators. In fact, the UVB lamp does not reduce to a minimum until $\sim 290 \mathrm{~nm}$, resulting in sizeable emissions below $295 \mathrm{~nm}$ which is reported to be the shortest wavelength found at the terrestrial level ${ }^{(6)}$. Thus due to the increased ability of UV radiation to elicit potentially damaging biological effects with decreasing wavelength, as demonstrated by the erythemal and DNA action spectra ${ }^{(18,19)}$, these spectroradiometric results suggest that the UVB fluorescent lamp is potentially the most hazardous irradiator under investigation.

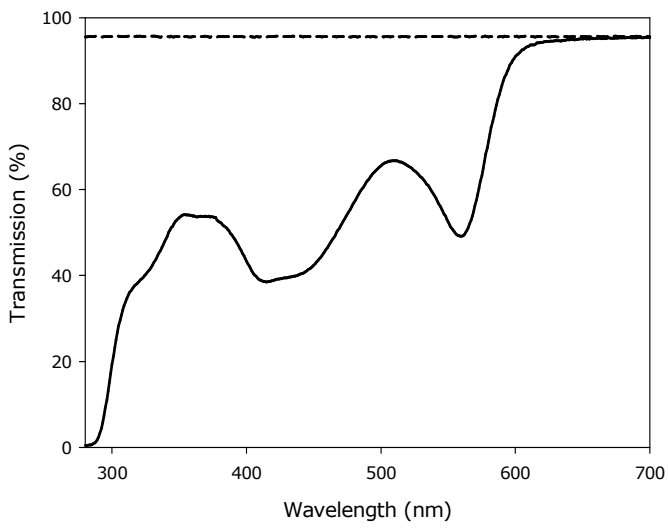

Figure 2 Transmission spectra of cell culture medium ) and PBS (ーーーー) measured using spectrally matched $1 \mathrm{~cm}$ quartz cuvettes in a dual beam PerkinElmer Lambda $900 \mathrm{UV} / \mathrm{Vis} / \mathrm{NIR}$ spectrometer, where the reference cuvette contained deionised water.
The primary media in which photo biological experimentation are performed are phosphate buffered saline (PBS) and cell culture medium, thus exposures were performed in both. Spectroscopic analysis of PBS and DMEM-F12 cell culture medium were measured using spectrally matched quartz cuvettes ( $1 \mathrm{~cm}$ optical path lengths) in a dual beam PerkinElmer Lambda 900 UV/Vis/NIR spectrometer to determine the potential attenuating effects of each exposure medium. The reference cuvette contained deionised water to minimise differences due to refraction at the interfaces. Figure 2 depicts the transmittance spectra for PBS and DMEM-F12 where PBS can be seen to have minimal losses in transmission while DMEM-F12 absorbs significantly below $600 \mathrm{~nm}$. The attenuating effects on the spectral distribution of each irradiator when transmitted through PBS and DMEM-F12 are depicted in figures $3 \mathrm{~A}$ and $3 \mathrm{~B}$ respectively. Computation of the spectral intensities of the modified spectra show DMEM-F12 to incur losses between $40-73 \%$ compared to near negligible losses of 4-5\% incurred by PBS as shown in table 2. These spectroscopic results suggest that PBS will result in increased cell death for a given exposure time due to minimal losses in transmission.
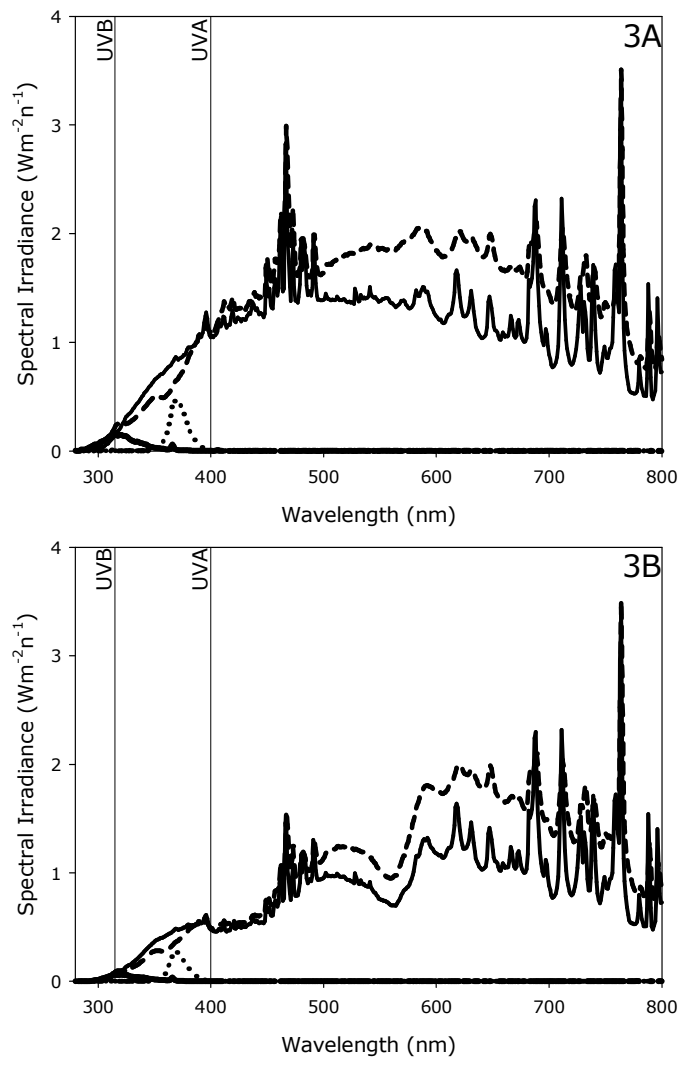

Figure 3 The irradiance spectra for the Q-sun solar simulator (-), the Oriel solar simulator operating at $35 \mathrm{~mA}(\boldsymbol{-} \boldsymbol{-})$, the UVA fluorescent lamp (י・י) and the UVB fluorescent lamp ( $)$ at their respective exposure levels when transmitted through PBS (3A) and DMEM-F12 cell culture medium (3B).

The delivery of each artificial source was sampled using a G5842 gallium arsenide phosphide (GaAsP) semiconductor photodiode (Hamamatsu photonics, 
Japan) in reverse bias with a frequency response of $3 \mathrm{kHz}$, a National Instruments DAQcard 700 and a LabVIEW $^{\mathrm{TM}}$ programme designed to act as an oscilloscope. Each irradiator was sampled in terms of voltage at a rate of 100,000 samples per second to prevent aliasing. The GaAsP photodiode has a response over the UV region only (figure 4) thus voltage measurements are indicative of current flow through the photodiode due to incident UV photons.

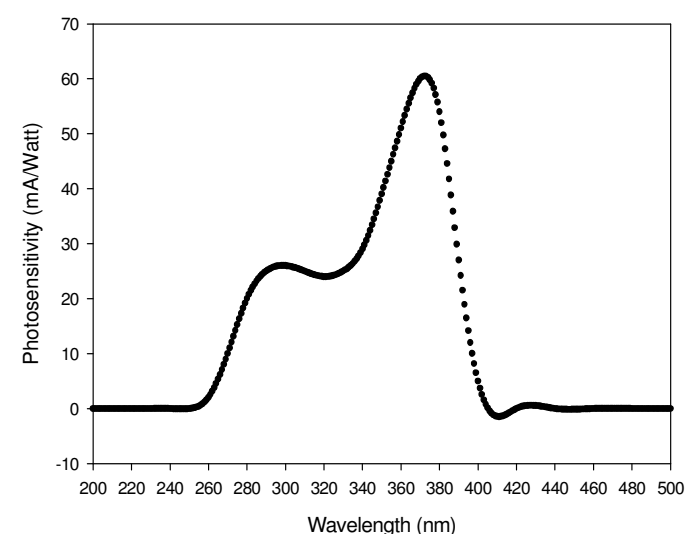

Figure 4 Spectral response curve for the Hamamatsu G5842 GaAsP photodiode

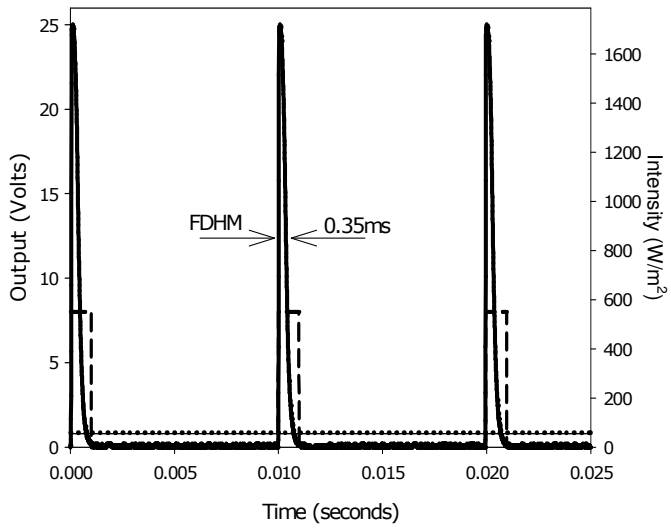

Figure 5 The pulsed output delivery of the Q-sun ( ), the FDHM continuous equivalent of the Q-sun $(\because \cdots)$, the continuous output of the Oriel ( and the ideal $100 \mathrm{~Hz}$ pulse with a $10 \%$ duty cycle of $1 \mathrm{~ms}$ $(\boldsymbol{-}-\boldsymbol{m})$.

The behaviour of each solar simulator is shown in figure 5. The Oriel was found to have a continuous output similar to solar radiation with a voltage output of $\sim 0.8$ Volts. The Q-sun, however, was found to be pulsed with a non variable frequency of $100 \mathrm{~Hz}$ and a peak output of 25 Volts per pulse. With a duty cycle of approximately $10 \%$ i.e. $1 \mathrm{~ms}$, it would be expected that the Q-sun voltage output be 10-fold higher than that of the Oriel giving an output of $\sim 8$ Volts, however as shown, this was not the case. The discrepancy between what is expected and what is observed is the difference between an ideal and a real pulsed source. An ideal source would pulse to 8 Volts for exactly $1 \mathrm{~ms}$ of its $10 \mathrm{~ms}$ cycle, also shown in figure 5 , thus yielding the same integral as would be expected from a constant source of 0.8 Volts. The Q-sun is not an ideal source and does not pulse to a maximum over the entire duty cycle thus the full duration at half maximum (FDHM) is considered. At FDHM the Q-sun pulse is approximately $1 / 29$ of the entire cycle $(0.35$ $\mathrm{msec} / 10 \mathrm{msec}$ ) where $1 / 29$ of 25 Volts yields a continuous equivalent of 0.86 Volts for the Q-sun which correlates well with the Oriel voltage output of 0.8 Volts. The voltage difference is attributed to differences in their spectral distribution in the UVA where the photodiode is maximally responsive.

Although the response of the photodiode is such that sources with different distribution spectra will incur different voltage outputs, the distribution of the Oriel and Q-sun are similar enough to allow an adjustment to determine an approximation on the UV irradiance delivered in a single Q-sun pulse. The simple adjustment employed was based on the fact that the Oriel UVA/B spectral irradiance of $\sim 55$ $\mathrm{Wm}^{-2}$ produced an output voltage of 0.8 Volts. From this a UV spectral irradiance in the region of $1600 \mathrm{Wm}^{-2}$ is estimated to be delivered in a single pulse from the Q-sun, an immense exposure that may be more detrimental to biological samples than an identical exposure from a continuous source.

Once the irradiators were fully characterised, each irradiator was employed to perform photo biological experiments as described in the methods section. The survival of HaCaT cells irradiated in either DMEM-F12 or PBS using the Q-sun and Oriel are shown in figures $6 \mathrm{~A}$ and $6 \mathrm{~B}$. It can be seen in figures $6 \mathrm{~A}$ and $6 \mathrm{~B}$ that minimal cell survival using the Q-sun was observed after exposures of 10 and 60 minutes in DMEM-F12 and PBS respectively. Similarly for the Oriel, 10 and 30 minute exposures in DMEM-F12 and PBS produced minimal cell survival, figures $6 \mathrm{~A}$ and $6 \mathrm{~B}$ respectively. The survival data in figure 6 is presented as a direct comparison of the DMEM-F12 and PBS dose response curves for each simulator, where the Oriel solar simulator can be seen to elicit increased cell killing, irrespective of exposure media, compared to identical exposures performed using the Q-sun solar simulator.

Exposures performed using the Q-sun solar simulator fall under the heading of flash photolysis due to the high frequency at which the lamp pulses $^{(21)}$. An initial concern using the Q-sun solar simulator was the possibility that the response of $\mathrm{HaCaT}$ cells would be rapid enough to discern the pulsed nature of the Q-sun and / or that the exaggerated dose administered during the duty cycle of the Q-sun would elicit responses different to those incurred by a continuous source. The BunsenRoscoe or reciprocity law states that all photochemical reactions are dependent on the total absorbed energy irrespective of the factors that determine the total dose i.e. irradiance and exposure time ${ }^{(20,21)}$. Although there are some reservations on the applicability of this law to biological systems due to the complex cellular responses to damage ${ }^{(22}$, $34)$, if the reciprocity law is obeyed then the same photo-response should be observed when the integral of the total dose administered is the same regardless of how the dose is delivered ${ }^{(21,35)}$. Comparisons of the dose response for HaCaT cells exposed using the Q-sun and Oriel show that the Q- 
sun results in increased survival compared to cells exposed under identical conditions using the Oriel regardless of exposure medium. This data shows that the Q-sun flash photolysis nature does in fact obey the reciprocity law, alleviating concerns regarding the environmental relevance of the Q-sun output delivery. The difference in cell survival observed between the two solar simulators is attributed to differences in their spectral intensities, specifically the UVB region where the Oriel solar simulator provides an output of $2.18 \mathrm{Wm}^{-2}$ compared to the Q-sun UVB output of $1.33 \mathrm{Wm}^{-2}$, and not behavioural differences. However, it is suspected that a critical or threshold frequency for biological irradiations exists since a decrease in frequency would require an increase in the irradiance delivered per pulse in order to achieve the same integral exposure. Thus, it is not unreasonable to believe that if the frequency of delivery is sufficiently reduced, an irradiance would be reached where the response of cells would begin to deviate significantly from those elicited under continuous irradiation conditions due to the immense insult administered per pulse and / or the response of the cells irradiated.
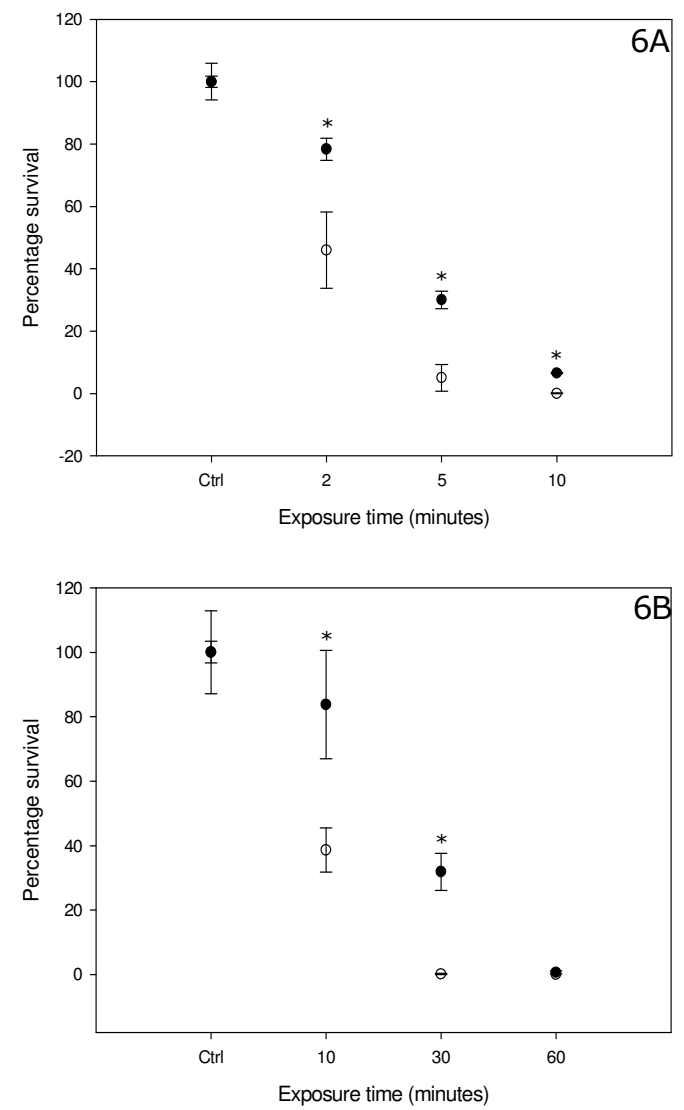

Figure 6 Direct exposure of $\mathrm{HaCaT}$ cells in DMEM-F12 $(6 \mathrm{~A})$ and PBS (6B) using the Q-sun $(\bullet)$ and the Oriel (O) solar simulators; $*$ represents a significant difference between survival elicited by the two solar simulators for a given exposure duration, $\mathrm{p} \leq 0.05$

A reciprocity study performed by Miyamoto et al (36) found that pulsed wave laser photodynamic therapy (PDT) and continuous wave PDT elicited apoptotic and necrotic deaths respectively. Based on the hypothesis that increased cytotoxicity is required to incur necrosis, Miyamoto et $\mathrm{al}^{(36)}$ concluded that pulsed exposures are less cytotoxic than continuous exposures.

Similarly to the work done by Miaymoto et $\mathrm{al}^{(36)}$, the results presented here show non continuous exposures to be less detrimental than continuous exposures. Although the differences observed in survival have been surmised to arise from differences in spectral output, there is the possibility that the simulators elicit different mechanisms of cell death. The clonogenic assay measures cell survival in response to some toxic event where little information regarding the mode(s) of cell death incurred (apoptosis or necrosis) can be extracted from the dose response curves. However, comparisons of the cell survival curves exposed in DMEM-F12 for both solar simulators (figure 6A) and similarly for cells exposed in PBS (figure 6B) show similar dose response curves. Suggesting that the solar simulators do not differ significantly, if at all, in their modes of cell death induced for a given exposure medium, however the possibility cannot be ruled out without further investigation.

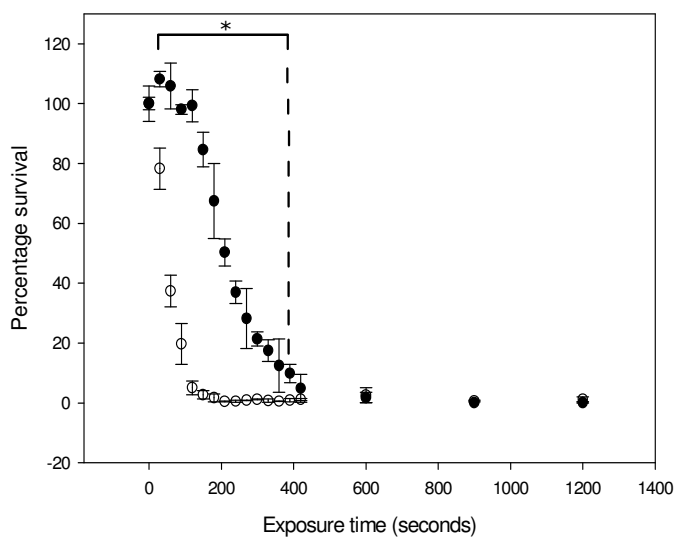

Figure 7 Direct exposure of HaCaT cells in DMEM-F12 cell culture medium $(\bullet)$ and PBS $(\mathbf{O})$ using the UVB fluorescent lamp, $*$ represents a significant difference between different exposure media for a given duration, $\mathrm{p} \leq$ 0.05

HaCaT cells irradiated using the UVB fluorescent lamp produced the most dramatic dose response curves (figure 7) of all irradiators under investigation. Cells irradiated in DMEM-F12 and PBS resulted in minimal survival after 7 and 3 minute exposures respectively. In contrast to solar simulator results, UVB lamp exposures performed in PBS and DMEM-F12 produced results that concur with the transmittance functions presented in figure 2, where PBS exposures resulted in increased cell killing compared to DMEM-F12 exposures. The UVB lamp has an irradiance of $2.43 \mathrm{Wm}^{-2}$ in the UVB region which is not exceptionally different to the UVB output of the Oriel which is $2.18 \mathrm{Wm}^{-2}$. However Oriel PBS exposures resulted in minimum survival after a 30 minute exposure whereas UVB lamp PBS exposures resulted in maximal cell killing within 3 minutes, a 10 fold difference in the tolerable exposure duration despite the lamps 
administering similar irradiance in the UVB. Revisiting the UV spectral distribution of both the Oriel and the UVB lamp in figure $1 \mathrm{~B}$, it can be seen that the photo biological results for the UVB lamp confirm the initial conjecture that the UVB lamp is the most hazardous irradiator owing to the sizeable and environmentally irrelevant emissions at wavelengths below $300 \mathrm{~nm}$. Thus due to the increasing efficacy of radiation with decreasing wavelength, it is clear that the UVB lamp provides a far greater biologically effective yet environmentally irrelevant irradiance, an effect that may have led to erroneous conclusions in the absence of spectroradiometry.

An intriguing effect regarding the exposure medium was revealed as a by-product of the reciprocity study performed. The spectroscopic results presented in figure 2 show that the irradiance and thus the dose received by a cell is greatly reduced for DMEM-F12 exposures compared to an identical exposure in PBS. Photo biological experiments performed using the UVB lamp concur with the spectroscopic results in figure 2. However, exposures carried out with both the Q-sun and Oriel solar simulators yield contradictory results where DMEM-F12 exposures have been shown to be far more effective than PBS exposures with regard to cell killing. A possible explanation for these conflicting results could be photosensitiser(s) present in DMEM-F12 cell culture medium whose absorption spectra may occur at longer less energetic wavelengths than those present in the UVB lamp spectral output. It is suspected that these hypothetically photosensitising wavelengths fall in the UVA waveband. Although the possibility of visible and infrared radiation effects, synergistic or otherwise, cannot be disregarded since the UVA lamp does not elicit cell death (data not shown). However this may be due to insufficient irradiance at, or omission of, said hypothetically photosensitising wavelengths in the spectral distribution of the UVA lamp. While the results presented in this paper support both reciprocity law compliance of the Q-sun and the importance of spectral distribution, it can also be concluded that DMEM-F12 augments the effects of solar simulated irradiation via some medium mediated effect. A full analysis of cell culture medium photosensitisation and its biological effects is currently under investigation with a corresponding publication in progress.

\section{CONCLUSIONS}

The importance of instrument calibration, spectral distribution and irradiance cannot be emphasised enough when attempting to discern the ability of solar radiation to elicit biological effects, as illustrated by the results presented in this paper.

Of particular interest with respect to the effects of spectral distribution, are the results obtained using the UVB lamp. The deleterious spectral distribution of the UVB irradiator is not obtrusively evident when compared to solar radiation, artificial and natural, yet its effect on survival was far more devastating than that elicited using solar simulated radiation. These effects may have been expected had the spectral irradiance of the UVB irradiator been weighted with an action spectrum such as the hazard spectrum [ref] which would clearly expose such detrimental capabilities. Furthermore, such spectral weighting could potentially predict the differential effects of two or more irradiators with seemingly similar spectral irradiances such as the solar simulators employed in this study. However, due to the rapidly increasing efficacy of action spectra with decreasing wavelength particularly in the UVB, weighting requires rigorous stray light rejection to minimise erroneous overestimations in irradiance particularly at biologically effective wavelengths such as those in the UVB. Stray light rejection is determined by the bandpass and hence optical resolution of a spectroradiometer ${ }^{(37)}$, which are nominally achieved with double monochromator spectroradiometers $^{(38)}$. Thus, despite stray light corrections outlined in the materials and methods section, the precision of data collected using a USB2000 spectrometer is not believed to be of a level at which weighting irradiance spectra would be appropriate. However, procurement of a bench top double monochromator spectroradiometer is planned to improve in house calibration techniques which will in turn facilitate spectral weighting. Thus, while it is important to acknowledge instrumental limitations, it is important to emphasise that data collected using a USB2000 spectrometer effectively serves its intended purpose which was to obtain reasonable approximations on the irradiance spectra of the irradiators under investigation.

These results demonstrate how even an apparently low output at sub terrestrial level wavelengths can significantly alter cellular responses. Furthermore, without comprehensive knowledge of the UVB lamp spectral distribution as provided using spectroradiometry, it may have been erroneously concluded that the augmented cell killing ability of the UVB irradiator was merely due to increased irradiance and not the presence of environmentally irrelevant wavelengths with enhanced biological efficacy.

The unexpected effects due to the exposure media not only demonstrates the importance of initial experimental parameters, but also highlight the implications of full spectrum irradiation. While it is desirable to know the contributions of individual wavebands, the possibility of interactions between different wavelengths present in solar radiation, whether the effects are synergistic, antagonistic, or purely additive, cannot be neglected and may yield significantly different results from individual waveband analysis. Although full spectrum irradiators can provide excellent approximations of solar radiation, it is not only the spectral distribution that determines the environmental relevance of an irradiator but also the manner in which the output is delivered.

Of the three irradiators for which results are presented, it was the non-continuous Q-sun solar 
simulator that was found to be the least phototoxic. Thus, it is concluded that the Q-sun is reciprocity law compliant and that both spectral distribution and irradiance are the principal attributes that dictate the biological efficacy of this irradiator despite the exaggerated manner in which the output of the Q-sun is delivered. However this may not hold true for all irradiators and is most probably frequency dependent and thus is yet another aspect of an artificial irradiator that may potentially confound photo biological experiments.

\section{ACKNOWLEDGEMENTS}

The authors are grateful to Dr. Petra Boukamp, DKFZ, Germany for the kind gift of the immortalised human keratinocyte $\mathrm{HaCaT}$ cell line. The authors would also like to thank Joe Condon of the Statistical Assistance Unit (SAU) in DIT for his valued advice and expertise in the statistical analysis of data.

\section{REFERENCES}

1. Gallagher, R. P. and Lee, T. K. Adverse effects of ultraviolet radiation: A brief review. Prog Biophys Mol Biol. 92, 119-131 (2006).

2. Reichrath, J. The challenge resulting from positive and negative effects of sunlight: How much solar UV exposure is appropriate to balance between risks of vitamin D deficiency and skin cancer? Prog Biophys Mol Biol. 92, 9-16 (2006).

3. Matsumura, Y. and Ananthaswamy, H. N. Toxic effects of ultraviolet radiation on the skin. Toxicol Appl Pharmacol. 195, 298-308 (2004).

4. Goukassian, D., Gad, F., Yaar, M., Eller, M. S., Nehal, U. S. and Gilchrest, B. A. Mechanisms and implications of the age-associated decrease in DNA repair capacity. Faseb J. 14, 1325-34 (2000).

5. de Gruijl, F. R., Sterenborg, H. J., Forbes, P. D., Davies, R. E., Cole, C., Kelfkens, G., van Weelden, H., Slaper, H. and van der Leun, J. C. Wavelength dependence of skin cancer induction by ultraviolet irradiation of albino hairless mice. Cancer Res. 53, 53-60 (1993).

6. Brown, D. B., Peritz, A. E., Mitchell, D. L., Chiarello, S., Uitto, J. and Gasparro, F. P. Common fluorescent sunlamps are an inappropriate substitute for sunlight. Photochem Photobiol. 72, 340-4 (2000).

7. Gróf, P., Rontó, G. and Sage, E. A computational study of physical and biological characterization of common UV sources and filters, and their relevance for substituting sunlight. J Photoch Photobio B. 68, 53-59 (2002).

8. Hanada, K. Photoprotective role of metallothionein in UV-injury -- metallothionein-null mouse exhibits reduced tolerance against ultraviolet-B. J Dermatol Sci. 23, S51-S56 (2000).

9. Ikehata, H., Kudo, H., Masuda, T. and Ono, T. UVA induces $\mathrm{C}->\mathrm{T}$ transitions at methyl-CpGassociated dipyrimidine sites in mouse skin epidermis more frequently than UVB. Mutagenesis. 18, 511-519 (2003).
10. Ikehata, H., Masuda, T., Sakata, H. and Ono, $\mathrm{T}$. Analysis of mutation spectra in UVB-exposed mouse skin epidermis and dermis: Frequent occurrence of C-T transition at methylated CpGassociated dipyrimidine sites. Environ $\mathrm{Mol}$ Mutagen. 41, 280-292 (2003).

11. Vink, A. A., Moodycliffe, A. M., Shreedhar, V., Ullrich, S. E., Roza, L., Yarosh, D. B. and Kripke, M. L. The inhibition of antigen-presenting activity of dendritic cells resulting from UV irradiation of murine skin is restored by in vitro photorepair of cyclobutane pyrimidine dimers. Proc Natl Acad Sci U S A. 94, 5255-60 (1997).

12. Katiyar, S. K. Interleukin- 12 and photocarcinogenesis. Toxicol Appl Pharmacol. 224, 220-227 (2007).

13. Nishigori, C. Cellular aspects of photocarcinogenesis. Photochem Photobiol Sci. 5, 208-14 (2006).

14. Melnikova, V. O. and Ananthaswamy, H. N. Cellular and molecular events leading to the development of skin cancer. Mutat Res Fund Mol M. 571, 91-106 (2005).

15. Terenetskaya, I. Spectral monitoring of biologically active solar UVB radiation using an in vitro model of vitamin $D$ synthesis. Talanta. 53, 195-203 (2000).

16. IRPA Proposed change to the IRPA 1985 guidelines on limits of exposure to ultraviolet radiation. International Non-ionizing Radiation Committee of the International Radiation Protection Association. Health Phys. 56, 971-2 (1989).

17. ICNIRP Guidelines on Limits of Exposure to Ultraviolet Radiation of Wavelengths between 180 $\mathrm{nm}$ and $400 \mathrm{~nm}$ (Incoherent Optical Radiation). Health Phys. 87, 171-186 (2004).

18. McKinlay, A. F. and Diffey, B. L. Reference action spectrum for ultraviolet induced erythema in human skin. CIE Journal. Volume 6, Pages 17-22 (1987).

19. Setlow, R. B. The wavelengths in sunlight effective in producing skin cancer: a theoretical analysis. Proc Natl Acad Sci U S A. 71, 3363-6 (1974).

20. Bunsen, R. and Roscoe, H. Photochemische untersuchungen. Ann Phys. 108, 193 (1859).

21. Martin, J. W., Chin, J. W. and Nguyen, T. Reciprocity law experiments in polymeric photodegradation: a critical review. Prog Org Coat. 47, 292-311 (2003).

22. Schindl, A., Rosado-Schlosser, B. and Trautinger, F. Reciprocity regulation in photobiology. An overview. Hautarzt. 52, 779-85 (2001).

23. Ocean-Optics (2008) Cosine-corrected Irradiance Probes

http://www.oceanoptics.com/Products/irradiancepro bes.asp 12 October 2009.

24. Walsh, J. E., Kavanagh, K. Y., Fennell, S., Murphy, J. and Harmey, M. Fibre-optic microspectrometers for biomedical sensing. T I Meas Control. 22, 355-369 (2000). 
25. Oliver, H. and Moseley, H. The use of diode array spectroradiometers for dosimetry in phototherapy. Phys Med Biol. 47, 4411 (2002).

26. International Commission on Illumination (CIE) (1987) International Lighting Vocabulary CIE pub. no. 17.4.

27. Boukamp, P., Petrussevska, R. T., Breitkreutz, D., Hornung, J., Markham, A. and Fusenig, N. E. Normal keratinization in a spontaneously immortalized aneuploid human keratinocyte cell line. J Cell Biol. 106, 761-71 (1988).

28. Lehman, T. A., Modali, R., Boukamp, P., Stanek, J., Bennett, W. P., Welsh, J. A., Metcalf, R. A., Stampfer, M. R., Fusenig, N., Rogan, E. M. et al. p53 mutations in human immortalized epithelial cell lines. Carcinogenesis. 14, 833-9 (1993).

29. Puck, T. T. and Marcus, P. I. Action of x-rays on mammalian cells. J Exp Med. 103, 653-66 (1956).

30. Diffey, B. L. Sources and measurement of ultraviolet radiation. Methods. 28, 4-13 (2002).

31. Gasparro, F. P. and Brown, D. B. Photobiology 102: UV sources and dosimetry - the proper use and measurement of "photons as a reagent". J Invest Dermatol. 114, 613-5 (2000).

32. Garland, C. F., Garland, F. C. and Gorham, E. D. Epidemiologic Evidence for Different Roles of Ultraviolet A and B Radiation in Melanoma Mortality Rates. Ann Epidemiol. 13, 395-404 (2003).
33. Bird, R. E. and Riordan, C. Simple Solar Spectral Model for Direct and Diffuse Irradiance on Horizontal and Tilted Planes at the Earth\&\#39;s Surface for Cloudless Atmospheres. J Appl Meteorol. 25, 87-97 (1986)

34. Merwald, H., Klosner, G., Kokesch, C., DerPetrossian, M., Hönigsmann, H. and Trautinger, F. UVA-induced oxidative damage and cytotoxicity depend on the mode of exposure. J Photoch Photobio B. 79, 197-207 (2005).

35. Sliney, D. H. Ultraviolet Radiation Effects Upon the Eye: Problems of Dosimetry. Radiat Prot Dosimetry. 72, 197-206 (1997).

36. Miyamoto, Y., Umebayashi, Y. and Nishisaka, $\mathrm{T}$. Comparison of phototoxicity mechanism between pulsed and continuous wave irradiation in photodynamic therapy. J Photoch Photobio B. 53, 53-59 (1999).

37. Weatherhead, E. C. and Webb, A. R. International Response to the Challenge of Measuring Solar Ultra-Violet Radiation. Radiat Prot Dosimetry. 72, 223-229 (1997).

38. Roy, C. R., Gies, H. P., Lugg, D. J., Toomey, S. and Tomlinson, D. W. The measurement of solar ultraviolet radiation. Mutat Res Fund Mol M. 422, 7-14 (1998). 
Table 1 UVA and UVB spectral irradiance for all sources, both artificial and natural. Solar radiation data for Albuquerque and Melbourne were obtained from the literature ${ }^{(30)}$ but all other data was sampled and calibrated using the Ocean Optics USB2000 spectrometer. The spectral irradiance data were then integrated to ascertain the UVA (315$400 \mathrm{~nm})$, UVB $(280-315 \mathrm{~nm})$ or the UVA and UVB $(280-400 \mathrm{~nm})$ irradiances for each irradiator at their respective exposure level i.e. where cells would be located during irradiation, while the Naples irradiance data correspond to measurements taken at ground level on a horizontal plain.

\begin{tabular}{|c|c|c|c|}
\hline Source/Location & UVA + UVB $\left(\mathrm{Wm}^{-2}\right)$ & $\mathrm{UVA}\left(\mathrm{Wm}^{-2}\right)$ & $\mathrm{UVB}\left(\mathrm{Wm}^{-2}\right)$ \\
\hline Qsun & 63.63 & 62.3 & 1.33 \\
\hline Oriel & 54.88 & 52.7 & 2.18 \\
\hline UVA lamp & 9.50 & 9.49 & 0.01 \\
\hline UVB lamp & 6.39 & 3.96 & 2.43 \\
\hline Naples & 46.52 & 45.25 & 1.27 \\
\hline Albuquerque & 51.93 & 50.28 & 1.65 \\
\hline Melbourne & 62.39 & 60.28 & 2.11 \\
\hline
\end{tabular}


Table 2 Losses incurred to the UVA-B spectral intensities of each irradiator when transmitted through PBS and DMEMF12 cell culture medium

\begin{tabular}{|c|c|c|c|c|c|c|c|}
\hline \multirow{2}{*}{ Light source } & \multirow[t]{2}{*}{ Media } & \multicolumn{2}{|c|}{ UVA \& UVB } & \multicolumn{2}{|c|}{ UVA } & \multicolumn{2}{|c|}{ UVB } \\
\hline & & $\left(\mathrm{W} / \mathrm{m}^{2}\right)$ & $\%$ loss & $\left(\mathrm{W} / \mathrm{m}^{2}\right)$ & $\%$ loss & $\left(\mathrm{W} / \mathrm{m}^{2}\right)$ & $\%$ loss \\
\hline \multirow[t]{3}{*}{ Qsun } & No media & \multicolumn{2}{|c|}{$63.63 \mathrm{~W} / \mathrm{m}^{2}$} & \multicolumn{2}{|c|}{$62.30 \mathrm{~W} / \mathrm{m}^{2}$} & \multicolumn{2}{|c|}{$1.33 \mathrm{~W} / \mathrm{m}^{2}$} \\
\hline & PBS & 60.84 & $-4 \%$ & 59.57 & $-4 \%$ & 1.27 & $-5 \%$ \\
\hline & DMEM-F12 & 31.56 & $-50 \%$ & 31.18 & $-50 \%$ & 0.38 & $-71 \%$ \\
\hline \multirow[t]{3}{*}{ Oriel } & No media & \multicolumn{2}{|c|}{$54.88 \mathrm{~W} / \mathrm{m}^{2}$} & \multicolumn{2}{|c|}{$52.70 \mathrm{~W} / \mathrm{m}^{2}$} & \multicolumn{2}{|c|}{$2.18 \mathrm{~W} / \mathrm{m}^{2}$} \\
\hline & PBS & 52.47 & $-4 \%$ & 50.39 & $-4 \%$ & 2.08 & $-5 \%$ \\
\hline & DMEM-F12 & 26.86 & $-51 \%$ & 26.21 & $-50 \%$ & 0.65 & $-70 \%$ \\
\hline \multirow[t]{3}{*}{ UVA lamp } & No media & \multicolumn{2}{|c|}{$9.50 \mathrm{~W} / \mathrm{m}^{2}$} & \multicolumn{2}{|c|}{$9.49 \mathrm{~W} / \mathrm{m}^{2}$} & \multicolumn{2}{|c|}{$0.01 \mathrm{~W} / \mathrm{m}^{2}$} \\
\hline & PBS & 9.09 & $-4 \%$ & 9.08 & $-4 \%$ & 0.01 & $-0 \%$ \\
\hline & DMEM-F12 & 5.06 & $-47 \%$ & 5.05 & $-47 \%$ & 0.006 & $-40 \%$ \\
\hline \multirow[t]{3}{*}{ UVB lamp } & No media & \multicolumn{2}{|c|}{$6.39 \mathrm{~W} / \mathrm{m}^{2}$} & \multicolumn{2}{|c|}{$3.96 \mathrm{~W} / \mathrm{m}^{2}$} & \multicolumn{2}{|c|}{$2.43 \mathrm{~W} / \mathrm{m}^{2}$} \\
\hline & PBS & 6.11 & $-4 \%$ & 3.78 & $-5 \%$ & 2.33 & $-4 \%$ \\
\hline & DMEM-F12 & 2.41 & $-62 \%$ & 1.75 & $-56 \%$ & 0.66 & $-73 \%$ \\
\hline
\end{tabular}

